\title{
PROGRESOS, RESTRICCIONES, ERRORES Y SORPRESAS EN CIRUGIA TUBARIA
}

\author{
Dr. Carlos D. Guerrero, F.A.C.S.
}

Mis primeros contactos con la cirugía tubaria fueron en 1936, siendo Residente del Sanatorio Español de México y ayudante del maestro Don Rosendo Amor; la técnica quirúrgica me fascinó, pero bien pronto los pobres resultados a la vez que me decepcionaron, fueron estímulo y razón para estudiar y meditar acerca del problema de la esterilidad conyugal y lograr para 1940, gracias a la comprensión del Director del mismo hospital, maestro Aguilar Alvarez, establecer el primer servicio en México equipado y orientado (1) al estudio y tratamiento de esterilidad.

En los años transcurridos desde entonces, he continuado en contacto permanente con este tipo de problemas y he podido apreciar que la cirugía tubaria ha sido motivo de atención, inquietud, controversia y discusión, habiendo ocupado muchas páginas de revistas y devoción especial en sesiones, congresos, reuniones, simposiums, cursos, etc. Ahora mismo en este VII Congreso Colombiano de Obstetricia y Ginecología, al cual he recibido el señalado honor de vuestra invitación, uno de los temas oficiales se refiere a Cirugía Tubaria.

He creído que la mejor manera de agradecer vuestra honrosa invitación, sea presentando un resumen de la experiencia acumulada en un tema sobre el cual todavía existen múltiples problemas por resolver.

\section{I - Progresos en cirugía tubaria}

La palabra "progreso" denota avance o mejoría, que a su vez se traduce en mejores resultados en cuanto a curación de un proceso patológico. En cirugía tubaria la valuación de los resultados se juzga por el restablecimiento de la permeabilidad, pero sobre todo por el logro de gestación intrauterina. A este respecto, los reportes que aparecen en la literatura mundial son absolutamente desconcertantes, yendo desde cifras de éxitos sorprendentes como son de hasta $90 \%$ de permeabilidades y $50 \%$ de gestaciones, hasta las cifras desalentadoras de la mayoría de los autores (2) y estos últimos resultados harían suponer que este tipo de cirugía estuviera en receso. Sin embargo, muy por el contrario, recuerdo varios conceptos que han variado en los últimos años de los que enumeraré los 10 siguientes: 1) Conocimiento de la patología tubaria susceptible de corrección quirúrgica, 2) Identificación de aquellas alteraciones en que la cirugía está contraindicada, 3) Requisitos, elementos y tiempo de preparación pre-operatoria de los casos seleccionados como favorables, 4) Determinación de la época apropiada para operar, 5) Universal aceptación 
de que la buena cirugía tubaria exige un aprendizaje específico y un entrenamiento mínimo, 6) Ensayo primeramente animal y humano posteriormente, de múltiples técnicas, materiales, artefactos, etc., con tendencia a conservar la permeabilidad, prevenir adherencias peritoneales y aún tratar de subsistir los oviductos, 7) Empleo de técnicas quirúrgicas cada vez más depuradas, 8) Seguir normas especiales en los cuidados postoperatorios inmediatos, 9) Control y cuidados reiterados a las operadas durante todo el primer año después de la intervención y 10) Tendencia a unificar criterios, técnicas, designación y valuación de los resultados.

Cada uno de estos puntos podría justificar un capítulo especial, pero congruente de la necesidad de limitarme al tiempo asignado, habré de referirme a algunos de los aspectos que no suelen ser abordados en los diversos libros $(3,4,5)$ o monografías $(6,7,8)$ a mi alcance.

\section{II - Restricciones de la Cirugía Tubaria}

Hace 20 años que B. B. Weinstein enumeró 10 condiciones para considerar indicada la cirugía tubaria y son:

1. La pareja desea profundamente tener un hijo.

2. El marido habrá de tener un semen con alto índice de fertilidad.

3. La mujer debe estar dentro de los límites de edad favorable para gestación.

4. Sin padecer ninguna enfermedad general que contraindique embarazo.

5. Teniendo un endometrio secretor comprobado en biopsias sucesivas.
6. Sin haber padecido recientemente un proceso pélvico inflamatorio.

7. Conservando un bajo índice de sedimentación hemática.

8. $Y$ este índice, conservado aún después de una palpación enérgica en pelvis.

9. Constituyendo un buen riesgo quirúrgico y más importante aún,

10. Constituir un buen riesgo mental.

Estos requisitos, no obstante el tiempo transcurrido, siguen vigentes, pero además deben respetarse algunas otras condiciones:

\section{A - Determinar sitio de obstrucción}

Puede situarse en el pabellón, en algún lugar a lo largo de la trompa - al nivel del cuerno. La histerografía es capital para establecer estas diferencias; sin embargo, en la actualidad nunca aceptamos como definitivo un diagnóstico radiológico de obstrucción sin ratificarle, antes y después de la radiografía, mediante la prueba de Rubin repetida.

\section{B - Fijar la clase de obstrucción}

Sin pretender hacer clasificación, únicamente enumeración, puedo mencionar: la obstrucción total o la simple estenosis o sub-obstrucción, en un solo sitio, en varios o todo lo largo de la trompa; incluyendo el cuerno uterino o dejando visible el esfínter; cuando están cerradas las fimbrias puede haber deformación, dilatación, angulación con o sin contenido líquido (hidro-salpinx). Por otro lado, la trompa en su luz puede estar sana, pero en cambio sufrir angulaciones, tracciones, elongaciones causadas por adherencias peritoneales o lesiones de órganos cercanos (miomas, quistes). 


\section{C - Sospechar el factor etiológico}

En principios de nuestra práctica clínica en este campo, la gonococcia era el factor predominante. En la actualidad esta entidad ha desaparecido casi y hemos aprendido a diagnosticar otros factores etiológicos como son: los congénitos, las obstrucciones post-aborto, las adherencias peritoneales post-operatorias, la endometriosis, la tuberculosis tubaria y las secuelas gonocócicas.

Una vez llenados los requisitos de Winstein y visto el tipo de obstrucción, se determinará si hay o no indicación quirúrgica. En la actualidad he aprendido a no operar en los siguientes casos: a) Si existe hipoplasia uterina. b) En sospecha de tuberculosis tubaria. c) En sospecha de endosalpingosis. y d) Cuando no se haya hecho previamente un tratamiento no quirúrgico, con insuflaciones e hidrotubaciones durante un tiempo razonable de 3 o 4 meses.

\section{III - Errores habituales de la cirugía tubaria}

Primer error. Consiste en no seguir fielmente las condiciones que restringen la indicación quirúrgica.

Segundo error. La falta de familiaridad con las técnicas quirúrgicas, recordando que las diferentes variedades de operaciones a nuestro alcance son:

\section{A) En la extremidad distal.}

- La desaglutinación de franjas o salpingolisis.

- La desobstrucción del pabellón, sacrificando algunas franjas o salpingo-ooforoplastía.

- Resección de una parte de la extremidad tubaria, haciendo un neopabellón (neo-salpingo ooforoplastía).
-Incisión longitudinal de trompa o salpingostomía.

B) En la porción ístmica de trompa.

La única operación lógica es la resección de la zona obstruída con neoimplantación al útero del cabo residual.

C) La cirugía en porción media de trompa, con salpingo-salpingo anastomosis (en la cual nunca he tenido éxito).

D) La cirugía extra-tubaria.

Se refiere a toda la cirugía en superficie peritoneal de trompa.

Tercer error. Olvidar algunos de los trucos quirúrgicos que contribuyen a facilitar la operación, prevenir re-obstrucciones y adherencias y evitar innecesarias mutilaciones.

Considero oportuno y útil transcribir a continuación dos decálogos que publiqué hace años.

\section{Lo que nunca hago:}

1. NUNCA operar con sospecha de infección latente en el cérvix, peritoneo o trompas.

2. NUNCA operar antes de tres meses después de haber practicado histerografía con medios oleosos. $Y$ NUNCA se hace la histerografía sin preguntar si existe predisposición o intolerancia al iodo. Con nuestra técnica de histerografía fue posible estudiar la dinámica tubaria e inyectar la mínima cantidad de contraste iodado.

3. NO usamos cánula intrauterina durante el acto operatorio porque al fijar el útero, se dificultan las maniobras sobre trompas.

4. NO TRACCIONAR las trompas violentamente, manipularlas lo menos 
posible y usar instrumental NO TRAUMATIZANTE.

5. NUNCA explorar la luz de las trompas con instrumentos rígidos y punzantes, capaces de herir la mucosa o dislacerarla, como estilete o sonda.

6. NUNCA mutilar total o parcialmente un anexo sin antes cerciorarse de que el otro está normal o menos lesionado.

7. $\mathrm{Si}$ es necesario resecar porciones de trompa, NUNCA dejar zonas dilatadas, porque ofrecen una zona fácil de embarazo ectópico.

8. NO dejar en la trompa nódulos palpables, pues son sugerentes de tuberculosis tubaria, endometriosis 0 salpingitis ístmica.

9. NO usar en el pabellón material de sutura o hemostasia capaz de causar reacción inflamatoria local, como catgut, membrana amniótica bovina, espuma de fibrina, etc.

10. NUNCA cerrar el abdomen sin cerciorarse de que la hemostasia fué perfecta.

Lo que creo recomendable hacer:

1. Las adherencias pélvicas las cortamos con tijera en vez de romperlas con maniobras digitales.

Una vez libre el útero (y las trompas) lo exteriorizamos con ayuda de tracción sobre los ligamentos redondos, o bien con dos cabos largos de catgut fuerte anudado en " $X$ " en pared posterior del útero.

2. Para explorar la permeabilidad de las trompas, del pabellón hacia el útero, usamos insuflación de aire, empleando jeringa de cristal, de punta cónica y roma y con bulbo de goma (asepto de B. D.).
También solemos introducir un delgado tubo de polietileno.

3. Para explorar la permeabilidad de útero hacia el pabellón, hacemos insuflación de aire o instilación de un líquido (antibiótico) o bien líquido y aire, con una jeringa común ( 10 cms.) a través de aguja de raquia introducida hasta cavidad uterina y obturando el istmo con una pinza de anillo.

4. Nos importa resecar la menor longitud de trompa, pero es más importante cuidar de dejar porción del órgano con buen calibre anatómico, con aspecto macroscópico sano y con permeabilidad normal previamente comprobado. Para suturas usamos seda o nylon 00000.

5. Para implantar la o las trompas, preferimos abrir ampliamente la cavidad uterina a través de una incisión transversal del fondo, lo que permite la inserción "a cielo abierto" y controlando la llegada hasta plena cavidad. No se necesita usar el reaming - sacabocado.

6. Para conducir la trompa hasta cavidad uterina, usamos el conductor maleable de Weisman, que también nos sirve para dejar el polietileno dentro de la trompa.

7. La trompa se sujeta procurando que no quede traccionada; el mesosálpinx se reseca y se fija al útero. El ovario se acerca al pabellón y se sujeta con algunas suturas en el meso-ovario.

8. Usamos el tubo de polietileno en los casos de implantación; indistintamente, uno de los cabos lo hacemos salir a vagina (con el conductor de Weisman) y lo suturamos al cérvix o bien lo dejamos en el tejido subcutáneo o asomado a la piel al nivel de la incisión de Pfanesthiel, que siempre ejecutamos. 
El tubo de polietileno se retira a los $2 \circ 3$ meses, después de una regla.

9. En los casos de plastía en pabellón, desde 24 horas después de la operación se inician las insuflaciones tubarias y se repiten diariamente por $5 \circ 6$ días. Reinsuflamos desde el siguiente mes.

En los casos de implantación, insuflamos hasta que retiramos el polietileno, $2 \circ 3$ meses después de operación y siempre al finalizar una regla.

10. Habitualmente usamos corticoides post-operatorios durante dos semanas, así como tetraciclinas y enzimas.

\section{IV - Sorpresas en la esterilidad de causa tubaria}

El último punto sobre el cual deseo llamar la atención de ustedes, se refiere a una serie de sorpresas que no es raro encontrar en los casos de esterilidad atribuída a obstrucción o anormalidad tubaria. Estas sorpresas pueden ser gratas 0 desagradables.

\section{Sorpresas gratas}

a) Falsa obstrucción tubaria.

Muchos pacientes llegan a nosotros con diagnóstico de obstrucción tubaria en ambos cuernos, hecho con una histerografía y solicitando ser operadas. En tales condiciones impongo mi apego a los requisitos recomendados y ya no nos sorprende que después de una buena preparación psíquica y farmacológica con una simple insuflación se obtenga una desobstrucción, seguida de gestación. Algunos de estos casos se explican por simple espasmo del esfínter del cuerno uterino.

b) Desobstrucción después de histerografía.
En otras ocasiones, la histerografía muestra obstrucción, la paciente es citada para hacer insuflación después de una nueva regla y con sorpresa se encuentra que tal menstruación falta y se presenta gestación. Obviamente que estas mujeres fueron curadas con la simple histerografía.

c) Desobstrucción medicamentosa sin operación.

Estoy convencido de la gran utilidad de las Ilamadas hidrotubaciones, recurso que usamos regularmente con la mezcla de: cloranfenicol, hidrocortisona y enzimas proteolíticas, como recurso pre-operatorio. De cada 10 mujeres buenas candidatas a cirugía tubaria, en quienes se aplica este recurso, logramos permeabilización tubaria en 4 y gestaciones en 2 (20\%). Téngase presente que las gestaciones con hidrotubaciones son más del $20 \%$, porque el procedimiento es además aplicado en muchos casos no buenos condidatos o con franca contraindicación a la cirugía.

d) Pseudo obstrucción tubaria demostrada durante la operación.

Siguiendo los requisitos repetidamente expuestos, para afirmar diagnóstico, indicamos y ejecutamos la operación y con sorpresa no encontramos las alteraciones que pudiéramos suponer.

Precisamente por lo posible de este grato error, una de nuestras rutinas transoperatorias recomendables es la confirmación de permeabilidad y la identificación de la zona obstruída.

\section{Sorpresas desagradables}

a) Inflamación pélvica iatrogénica. Al seguir una rutina diagnóstica de esterilidad o al seguir el proceso de requisitos exigibles para aceptar hacer una operación, debemos hacer 
una insuflación y una histerografía. Hemos tenido la muy desagradable experiencia de que no obstante las precauciones, después de algunos de estos recursos se presenta la agudización de un proceso tubario séptico larvado, que obviamente deja una secuela en que la trompa queda en peores condiciones que antes estuviera. Creemos que la mejor prevención de esta complicación sea la delicadeza en las maniobras y la exclusión de un foco séptico endocervical o tubario y la ejecución del procedimiento precisamente post-menstrual. nes.

b) Intolerancia a las hidrotubacio-

He tenido dos casos en que se despertó dolor severo y aún reacción peritoneal aguda, con estado de colapso, durante la ejecución de hidrotubación. En ambos casos la suspensión del recurso, el reposo y sedación fueron suficientes para curar y por cierto que ambos casos, sorpresivamente fueron seguidos de gestación.

También he observado reacciones peritoneales en 4 casos; pero uno de ellos seguido de gestación.

Creo que estas reacciones a la hidrotubación demuestran precisamente que el proceder logra desobstruir la trompa. cada.

c) Patología tubaria no diagnosti-

La operación se solicita con un diagnóstico de obstrucción y el programa quirúrgico anhela hacer una reparación. Algunas veces nos encontramos con lesiones absolutamente irreparables, en las cuales es preferible hacer una mutilación.

d) Patología tubaria erróneamente conservada.
Algunas veces con el anhelo de conservar "algo" de trompa y con "alguna permeabilidad", podemos estar dejando una trompa, de tal suerte que en meses después afrontemos la sorpresa de una gestación ectópica.

\section{Consideraciones finales}

Podría haber traído a ustedes un trabajo, como algunos publicados elaborado con las normas exigidas en Estados Unidos a saber: razón del estudio, material, métodos, discusión y conclusiones, pero he preferido traerles un juicio sincero, fundamentado en treinta años de experiencia en esterilidad y en poco más de 400 intervenciones en trompas; este número de casos no es exagerado, apenas representa un promedio de 20 casos al año en los últimos 20 años.

La cirugía tubaria es benigna; pero por desgracia el resultado postoperatorio es con frecuencia decepcionante y la patología iatrogénica post-operatoria es común y es por esta consideración, que tanto he insistido en la cuidadosa selección del caso por operar.

Las gestaciones intrauterinas logradas en mis casos alcanzan porcentajes modestos, apenas $10 \%$ en casos de cirugía de pabellón, $24 \%$ en las neoimplantaciones y $45 \%$ en cirugía del peritoneo tubario. Es en consideración a este bajo índice de éxito, que debemos estar obligados de advertir a toda mujer por operar, el pronóstico; después de nuestra explicación, algunas mujeres cancelan la operación, otras la aceptan y éstas últimas se encuentran ampliamente retribuídas al obtener un embarazo, pero además al no obtener embarazo, psicológicamente tranquilas, porque saben que han puesto de su parte todo lo posible para lograr su ansia de maternidad o al menos para demos- 
trarle a un marido egoista, que ella ofreció todo su sacrificio para complacerlo.

Mientras más años transcurren, menos cirugía tubaria practico, porque cada vez escojo más mis casos, cada vez aplico más recursos médicos, cada vez sé esperar más y cada vez también, frente a cada operación de trompas, siento la angustia de ignorar si podré o no ayudar a una pobre mujer estéril que está concentrando toda su esperanza en dicha operación.

Pero ello no quiere decir que la cirugía tubaria deba abandonarse, porque sigue siendo un recurso, que en el caso bien seleccionado, es el único posible.

\section{BIBLIOGRAFIA}

AMERICAN MEDICAL ASSOCIATION: Standard Nomenclatura of Diseases and Operations. Fifth edition. 3-81, 105-482, 484-510, 517 610. The Blackiston Company, New York, Philadelphia, Toronto.

LOPEZ-PICHOURON RAFAEL: La Estadística en el Hospital Colombiano Moderno. Tesis Universidad Javeriana. Bogotá, octubre, 1962.
LOPEZ-RUIZ RAFAEL: Bioestadística simplificada y Código Internacional O.M.S. (resumido). Revista del Hospital San Juan de Dios, Bogotá (II), 8: 324-329. Año 1954. Auge Científico-Asistencial en los Establecimientos de San Juan de Dios. 33: 40, Vol. VI, No $24,1962$.

LOPEZ-RUIZ RAFAEL: Morbilidad y mortalidad en los Establecimientos Hospitalarios de San Juan de Dios. Bogotá, 1960, 62, 63. Tribuna Médica (Vol. IV), No 164, Noviembre, 1964. Tribuna Médica (Vol. V), № 209, Octubre, 1965. Tribuna Médica (Vol. VI), No 257. Octubre 3, 1966. Tribuna Médica (Vol. VI) No 295, Julio 10, 1967. Ediciones Lerner, Avenida Jiménez Nọ 4-33, Bogotá.

LOPEZ-RUIZ RAFAEL: Morbilidad y Mortalidad en el Instituto Materno Infantil "Concepción Villaveces de Acosta" durante los años 1960, $61,62,63$ y 64 . Revista de Obstetricia y Ginecología. (Vol. XVI), No 4: 312-349, Julio, Agosto, 1965. Ediciones Lerner. Avenida Jiménez Nọ 4-33. Bogotá.

ORGANIZACION MUNDIAL DE LA SALUD (O. M. S.). Manual de Clasificación Estadstica Internacional de Enfermedades y Causas de Defunción. Revisión 7/55, 3-43, 48-316, Ginebra, Mayo, 1957. Indice de diagnósticos de hospitales y clasificación de operaciones; 9$211,212-273$. Publicaciones Científicas № 52, Washington, D. C., Mayo, 1961. 